\title{
INTERAÇÃO DE PLANTAS MEDICINAIS COM ANTI-HIPERTENSIVOS
}

\author{
INTERACTION OF MEDICINAL PLANTS WITH ANTIHYPERTENSIVES
}

\author{
Ana Clara Duarte de Oliveira ${ }^{1}$ \\ Thiago Oliveira da Silva Barreto ${ }^{2}$ \\ José Fernando de Araújo Neto ${ }^{3}$
}

RESUMO: O objetivo deste estudo é realizar uma revisão de literatura sobre as implicações do uso de plantas medicinais no contexto da hipertensão arterial. Esse estudo possui natureza qualitativa, descritiva e exploratória. Para a pesquisa foi realizada uma revisão da literatura científica. $\quad \mathrm{Na}$ revisão bibliográfica realizada, foram encontrados 68 artigos. Dentre os artigos encontrados, 25 enquadraram-se nos critérios de inclusão. Os demais foram desconsiderados, pois não abordavam sobre o uso de plantas medicinais poderiam causar alteração na pressão arterial e as possíveis interações com anti-hipertensivos. Com o trabalho foi possível concluir que as plantas medicinais utilizadas de forma irracional, sem a orientação de um profissional habilitado, acarretam risco à saúde, e podem propiciar a ocorrência de eventos adversos e interações medicamentosas negativas quando utilizados concomitantemente com outros medicamentos.

Palavras-chave: Plantas Medicinais. Uso racional de plantas medicinas. Orientação Farmacêutica.

ABSTRACT: The aim of this study is to conduct a literature review on the implications of the use of medicinal plants in the context of arterial hypertension. This study has a qualitative, descriptive and exploratory nature. For the research, a review of the scientific literature was carried out. In the bibliographic review, 68 articles were found. Among the articles found, 25 met the inclusion criteria. The others were disregarded, as they did not address the use of medicinal plants that could cause changes in blood pressure and possible interactions with antihypertensive drugs. With the work it was possible to conclude that medicinal plants used irrationally, without the guidance of a qualified professional, pose a risk to health, and can lead to the occurrence of adverse events and negative drug interactions when used concomitantly with other medications.

\footnotetext{
I Graduanda em farmácia na universidade salvador (Unifac). E-mail: anaclaraduarter6@hotmail.com

2Graduando em farmácia na universidade salvador (Unifacs).E-mail: thiagobarreto493@gmail.com.

3 Possui graduação em Farmácia pela Universidade Federal da Bahia (2013) e Mestrado em Química pela Universidade Federal da Bahia (2016). Atualmente cursa o Doutorado em Química pela Universidade Federal da Bahia. Tem experiência na área de Química, com ênfase em Química dos Produtos Naturais, atuando principalmente nos seguintes temas: Vismia, Erythroxylum, citotoxicidade, screening fitoquímico, atividade antioxidante, acetilcolinesterase e antimicrobiana. E-mail: jfernandoaraujo@hotmail.com.
} 
Keywords: Medicinal Plants. Rational use of medicinal plants, Pharmaceutical Guidance.

\section{INTRODUÇÃO}

O uso plantas medicinais para o tratamento ou cura de uma doença não é algo recente. A terapia com plantas em algumas situações acaba sendo a única estratégia para o enfrentamento dos problemas de saúde (BADKE, 2012).

De acordo com Maciel et al., (2002), nos últimos anos está acontecendo um aumento progressivo no uso de ervas medicinais pela população do mundo inteiro. Esse aumento guarda relação com o aparecimento de patologias não tratáveis adequadamente, pela disseminação da ideia de que as plantas por serem de origem vegetal não apresentam nocividade a vida das pessoas, sendo mais seguras que fármacos sintéticos, outro fator que pode estar relacionado ao aumento do consumo das ervas e a deficiência no acesso aos serviços de saúde (MACIEL et al., 2012).

Dentre as patologias que os pacientes mais recorrem, destacamos a Hipertensão Arterial Sistêmica que tem sido apontada como um grave problema de saúde pública e que de acordo a VI Diretrizes Brasileira de hipertensão (2oro) é uma condição clínica multifatorial, que é caracterizada por níveis elevados e sustentados da pressão arterial com associação de transformações na função dos órgãos afetados (rins, vasos sanguíneos, coração e encéfalo). Assim como as alterações metabólicas, modificações no volume do líquido circulante e resistência vascular periférica resulta em riscos cardiovasculares.

Vale destacar, segundo Nogueira (2014) que para o controle da Hipertensão Arterial Sistêmica, além do tratamento medicamentoso, é necessário que o indivíduo abandone

hábitos de vida que as deixe ainda mais vulneráveis a elevação de níveis pressóricos, como a ingesta em demasia de sódio, o sedentarismo, o tabagismo e o etilismo.

Em se tratando das terapias alternativas, Dias (2009) destaca o uso de plantas medicinais, advertindo que a utilização das ervas medicinais concomitantemente com medicamentos pode ocasionar efeitos negativos, muitas vezes, justificadas pela falta de informação sobre as substâncias, além da mitificação de que produtos de origem vegetal não causam danos a saúde, podendo ser utilizados por qualquer pessoa.

No que diz respeito a nocividade das plantas medicinais para a saúde, Castro (2006) elenca que os problemas mais prevalentes são as intoxicações, as reações alérgicas, as interações medicamentosas. $\mathrm{O}$ autor destaca que a ocorrência desses efeitos pode ser ainda potencializada pelo equívoco em identificar as espécies consumidas, bem como a forma que as ervas são cultivadas, colhidas, estocadas, conservadas e produzidas.

No que concerne a justificativa para realização deste estudo, sabe-se que a hipertensão arterial sistêmica, dentre as doenças crônicas, consiste em um dos problemas de saúde de maior magnitude na atualidade, sendo uma das principais causas de morbidade e mortalidade cardiovascular no Brasil. O manejo da HAS consiste no uso de medicamentos convencionais e plantas medicinais, essa associação cada vez mais comum, amplificando os riscos de ocorrência de interações (RAWAT,2016).

Entre as formas de tratamento da HAS, a VII Diretriz Brasileira de Hipertensão Arterial, citam duas formas: o tratamento não medicamentoso (TNM) da HAS, que envolve controle ponderal, medidas nutricionais, prática de atividades físicas, cessação do 
tabagismo, controle de estresse e o tratamento medicamentoso da que visa, em última análise, a redução da morbimortalidade Cardiovascular.

Deste modo, o presente estudo justifica-se pela intenção de abordar um tema que poderá auxiliar o profissional farmacêutico a garantir segurança do uso de plantas medicinais, promovendo o uso racional através do processo de orientação. Sendo o profissional farmacêutico importante na difusão de informações relativas as terapias medicamentosas no contexto da hipertensão, bem como as implicações no uso concomitante de medicamentos anti-hipertensivos com plantas medicinais, tornam-se relevante a realização de estudos sobre este tema pela intencionalidade de levar à comunidade acadêmica informações sobre o assunto.

Para tanto traçou-se como objetivo deste estudo: realizar uma revisão de literatura acerca das implicações do uso de plantas medicinais no contexto da hipertensão arterial.

\section{METODOLOGIA}

Trata-se de uma revisão de literatura, de natureza qualitativa, descritiva e exploratória. Para a pesquisa foi realizada uma revisão da literatura científica. Neste estudo, foram utilizados artigos pesquisados nas principais bases de dados, Scielo, BDENF, LILACS, MEDLINE. Para realizar a busca foram utilizados os seguintes descritores: "Uso racional" e "plantas medicinais", "Plantas" e "Anti-hipertensivo" e seus correspondentes em inglês, "Rational use" and "medicinal plants", "Plants" and "antihypertensive". Foram utilizados também para a busca de artigos científicos as seguintes palavras chaves: Plantas medicinais, Assistência Farmacêutica e Uso racional de plantas medicinas. Os artigos selecionados seguiram os seguintes critérios de inclusão: publicados entre 2004 e 2020 nos idiomas inglês e português, disponíveis gratuitamente para consulta. Os critérios de exclusão utilizados foram: artigos repetidos, artigos de revisão, aqueles que não atenderem a temática e os que não foram publicados durante o período delimitado.

\section{RESULTADOS E DISCUSSÃO}

$\mathrm{Na}$ revisão bibliográfica realizada, foram encontrados 68 artigos. Dentre os artigos encontrados, 25 enquadraram-se nos critérios de inclusão. Os demais foram desconsiderados, pois apesar de aparecerem na busca realizada nas bases eletrônicas, não abordavam sobre que plantas medicinais poderiam causar alteração na pressão arterial e interação com antihipertensivo.

De acordo com os artigos selecionados neste levantamento bibliográfico, 8 plantas (Catha edulis, Ginkgo biloba L., Pausinystalia Yohimbe, Ruscus aculeatus, Plectranthus barbatus Andr. Baccharis trimera, Camellia sinensis (L.) Kuntze., Hypericum perforatum) mostraram-se alterando os níveis pressóricos e interagiam com medicamentos usados para controle da pressão arterial sistêmica.O Quadro I, mostra caraterísticas das plantas medicinais que acarretam danos à saúde quando utilizadas em associação com os anti-hipertensivos. 
Quadror. Características de plantas medicinais que acarretam danos a saúde quando utilizadas em associação com anti-hipertensivos.

\begin{tabular}{|c|c|c|c|c|}
\hline $\begin{array}{l}\text { PLANTA } \\
\text { MEDICINA } \\
\text { L }\end{array}$ & COMPOSIÇÃO QUÍMICA & INDICAÇÕES & PRINCIPAL RISCO & $\begin{array}{l}\text { AUTORES E ANO } \\
\text { DE PUBLICAÇÃO }\end{array}$ \\
\hline $\begin{array}{l}\text { Catha edulis } \\
\text { (Flor do } \\
\text { paraíso) }\end{array}$ & $\begin{array}{l}\text { Alcaloides, Flavonoides (cardamonin, isalpinin } \\
\text { etc.), catequina, epicatequina, óleos essenciais } \\
\text { (canfeno, cânfora etc.), rutina e dois derivados } \\
\text { glicosídicos do kaempferol, taninos. }\end{array}$ & $\begin{array}{l}\text { Afecção da pele, artrite, } \\
\text { asma, catarro, cistite, micose } \\
\text { de pele, pelos e unhas. }\end{array}$ & Aborto, hipertensão. & $\begin{array}{l}\text { ROCHA (2004), } \\
\text { NOGUEIRA (2014) } \\
\text { ROSARIO et al., } \\
\text { (2009), } \\
\text { SILVEIRA (2008) } \\
\text { AHMAD (2015) } \\
\text { RAWAT (2016) }\end{array}$ \\
\hline $\begin{array}{l}\text { Ginkgo biloba } \\
\text { L. } \\
\text { (Ginkgo } \\
\text { biloba) }\end{array}$ & $\begin{array}{l}\text { Ácido butanóico, ácido ginkgólico, ácidos } \\
\text { graxos, alcanos, antocianina, ginkgetina, } \\
\text { benzenóides, bioflavonoides, canferol, } \\
\text { carboidratos, carotenóides, catequina, } \\
\text { diterpenos ginkgolídeos A, B, C, J e M, ésteres } \\
\text { de ácido cumárico, esteróis, fenilpropanóides, } \\
\text { ginol, glicosídeos flavonoides (principalmente } \\
\text { ginkgobilina, quercetina e isoramnetina), } \\
\text { kaempferol, lactona bilobalida, lipídeos, } \\
\text { minerais, quercetina, sitosterol, triterpenos. } \\
\text { Frutos: ácidos ginkgólicos, ginol. }\end{array}$ & $\begin{array}{l}\text { Tratamento de distúrbios de } \\
\text { memória e concentração, } \\
\text { vertigens, zunido no ouvido e } \\
\text { labirintite. }\end{array}$ & $\begin{array}{l}\text { Distúrbios, } \\
\text { circulatório, } \\
\text { hipertensão. }\end{array}$ & $\begin{array}{l}\text { ROCHA (2004) } \\
\text { NOGUEIRA (2014) } \\
\text { ROSARIO et al., } \\
\text { (2009) } \\
\text { SILVEIRA (2008) } \\
\text { AHMAD (2015) } \\
\text { RAWAT (2016) }\end{array}$ \\
\hline
\end{tabular}




\begin{tabular}{|c|c|c|c|c|}
\hline $\begin{array}{l}\text { Pausinystalia } \\
\text { Yohimbe } \\
\text { (Casca de } \\
\text { ioimba, } \\
\text { ioimbé) }\end{array}$ & Yohimbina (afrodina), Yohimbina, ajmalina. & $\begin{array}{l}\text { Impotência erétil, frigidez, } \\
\text { fadiga em portadores de } \\
\text { HIV, obesidade, febre, tosse, } \\
\text { lepra. }\end{array}$ & $\begin{array}{l}\text { Paralisia muscular, } \\
\text { hipertensão. }\end{array}$ & 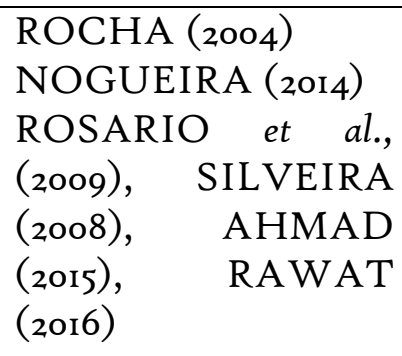 \\
\hline $\begin{array}{l}\text { Ruscus } \\
\text { aculeatus } \\
\text { (Gilbarbeira) }\end{array}$ & $\begin{array}{l}\text { Cálcio, óleo essencial, flavonóides, potássio, } \\
\text { resina, sódio. }\end{array}$ & $\begin{array}{l}\text { Cansaço, menopausa, } \\
\text { insuficiência venosa e } \\
\text { renal. }\end{array}$ & $\begin{array}{l}\text { Hipertensão, } \\
\text { intolerância gástrica. }\end{array}$ & $\begin{array}{l}\text { ROCHA (2004), } \\
\text { NOGUEIRA (2014) } \\
\text { ROSARIO et al., } \\
\text { (2009), } \\
\text { SILVEIRA (2008), } \\
\text { AHMAD (2015), } \\
\text { RAWAT (2016) }\end{array}$ \\
\hline $\begin{array}{l}\text { Plectranthus } \\
\text { barbatus } \\
\text { Andr. } \\
\text { (Falso Boldo) }\end{array}$ & $\begin{array}{l}\text { Óleo essencial (guaieno e fenchona), barbatol, } \\
\text { barbatesina, cariocal, barbatusol, forskolina }\end{array}$ & $\begin{array}{l}\text { Distúrbios da digestão: dor } \\
\text { de barriga principalmente se } \\
\text { acompanhada de espasmos, } \\
\text { náuseas, úlcera e dores de } \\
\text { estômago (azias). Males } \\
\text { hepáticos (problemas do } \\
\text { fígado). Gripe, resfriado,- } \\
\text { Tosse. }\end{array}$ & $\begin{array}{l}\text { Hipertensão, gastrite, } \\
\text { úlcera. }\end{array}$ & $\begin{array}{l}\text { ROCHA (2004) } \\
\text { NOGUEIRA (2014) } \\
\text { ROSARIO et al., } \\
\text { (2009), } \\
\text { SILVEIRA (2008), } \\
\text { AHMAD (2015), } \\
\text { RAWAT (2016) }\end{array}$ \\
\hline
\end{tabular}


Revista Ibero- Americana de Humanidades, Ciências e Educação- REASE $\quad$ open $\mathbf{O}$ access

\begin{tabular}{|c|c|c|c|c|}
\hline $\begin{array}{l}\text { Baccharis } \\
\text { trimera } \\
\text { (Carqueja) }\end{array}$ & $\begin{array}{l}\text { Alfa e beta-pineno, álcoois sesquiterpênicos, } \\
\text { ésteres terpênicos, flavonas, flavanonas, } \\
\text { saponinas, flavonóides, fenólicos, lactonas } \\
\text { sesquiterpênicas e tricotecenos, alcalóides. }\end{array}$ & $\begin{array}{l}\text { Febre, reumatismo, } \\
\text { Asma, Azia. }\end{array}$ & Hipertensão, aborto. & $\begin{array}{l}\text { ROCHA (2004) } \\
\text { NOGUEIRA (2014) } \\
\text { ROSARIO et al., } \\
\text { (2009), } \\
\text { SILVEIRA (2008) } \\
\text { AHMAD (2015) } \\
\text { RAWAT (2016) }\end{array}$ \\
\hline $\begin{array}{l}\text { Camellia } \\
\text { sinensis (L.) } \\
\text { Kuntze. } \\
\text { (Chá-verde) }\end{array}$ & $\begin{array}{l}\text { flúor, catecinas, alcalóides, óleo essencial, } \\
\text { bioflavanóides, teogalina, ácido ascacórbico, } \\
\text { ácido gálico, ácido quinico, betacaroteno, } \\
\left.\text { tiamina (vitamina } \mathrm{B}_{\mathrm{I}} \text { ), riboflavina ( } \mathrm{B} 2\right) \text {, } \\
\text { vitamina } \mathrm{C} \text {, vitamina B6, ácido } \\
\text { fólicotheanina, metixantinas, cafeína, } \\
\text { carboidratos, tanino, minerais }\end{array}$ & $\begin{array}{l}\text { Anti-cancerígena, } \\
\text { obesidade, doenças } \\
\text { cardiosvasculares. }\end{array}$ & $\begin{array}{l}\text { Pode } \\
\text { hiperacidez, redução } \\
\text { do apetite, prisão de } \\
\text { ventre ou diarréia e } \\
\text { irritação do aparelho } \\
\text { digestivo. }\end{array}$ & $\begin{array}{l}\text { Resolução RE n. } 89, \\
\text { de i6 de março de } 2004\end{array}$ \\
\hline $\begin{array}{l}\text { Hypericum } \\
\text { perforatum } \\
\text { (Erva-de- } \\
\text { são-joão) }\end{array}$ & $\begin{array}{l}\text { ácido chiquimico, biflavonas, catequinas, } \\
\text { carotenos, derivados fluoroglucinólicos, } \\
\text { fitosterois (beta-sitosterol), flavonóides } \\
\text { (hiperosídeo, rutina, quercetina), } \\
\text { glicosídeos (hipericina, corante vermelho), } \\
\text { hiperforina, pectinas, a-pineno, princípios } \\
\text { amargos, procianidinas, pseudohipericina, } \\
\text { resinas, saponina, taninos, terpineol, } \\
\text { vitamina C e P. }\end{array}$ & $\begin{array}{l}\text { acidez estomacal, acne, } \\
\text { afecções pulmonares, } \\
\text { afecções das vias urinárias, } \\
\text { caspa, depressão, diarréias } \\
\text { crônicas, } \\
\text { pulmonares, doenças } \\
\text { musculares causadas por } \\
\text { contusões e excesso de } \\
\text { atividade física, enurese } \\
\text { noturna, feridas, gota, } \\
\text { hemorroidas, insuficiência }\end{array}$ & $\begin{array}{l}\text { Pode interferir nos } \\
\text { efeitos de drogas } \\
\text { contraceptivas. } \\
\text { Gestantes e usuárias de } \\
\text { contraceptivos } \\
\text { (anticoncepcionais), } \\
\text { degrem evitar seu uso. }\end{array}$ & $\begin{array}{l}\text { Resolução RE n. } 89 \text {, } \\
\text { de I6 de março de } 2004\end{array}$ \\
\hline
\end{tabular}




\begin{tabular}{|l|l|l|}
\hline & $\begin{array}{l}\text { hepática, enurese infantil, } \\
\text { insônia, má digestão, mau } \\
\text { funcionamento do fígado e } \\
\text { dos rins, nervosismo, } \\
\text { queimaduras de primeiro } \\
\text { grau, reumatismo, seborréia, } \\
\text { ulcerações internas, vermes } \\
\text { intestinais }\end{array}$ \\
\hline
\end{tabular}

Fonte: dados da pesquisa (2021)

A fim de dinamizar a análise sobre as implicações do uso concomitante de plantas medicinais com as classes de medicamentos antihipertensivo, segue o Quadro 2 com o nome científico, o nome popular, a ação das plantas medicinais sob as classes de anti-hipertensivos e os autores que produziram estudos sobre a temática e ano de publicação dos respectivos estudos. O levantamento da bibliografia referente a temática, foi feita através do nome científico, para a garantia de informações confiáveis, visto que uma erva pode apresentar vários nomes populares a depender das regiões.

Quadro 2. Ação das plantas medicinais sob as classes de anti-hipertensivos

\begin{tabular}{|l|l|l|l|l|}
\hline $\begin{array}{l}\text { NOME } \\
\text { CIENTÍfICO }\end{array}$ & $\begin{array}{l}\text { NOME } \\
\text { POPULAR }\end{array}$ & $\begin{array}{l}\text { AÇÃO SOBRE } \\
\text { MEDICAMENT } \\
\text { O ANTI- } \\
\text { HIPERTENSIVO }\end{array}$ & $\begin{array}{l}\text { CLASSE DE ANTI-HIPERTENSIVOS QUE } \\
\text { INTERAGE }\end{array}$ & $\begin{array}{l}\text { AUTORES E ANO DE } \\
\text { PUBLICAÇÃO }\end{array}$ \\
\hline Catha edulis & $\begin{array}{l}\text { Flor do } \\
\text { paraíso }\end{array}$ & $\begin{array}{l}\text { Antagonista } \\
\text { Pampaticolíticos } \\
\text { Exemplos: metildopa, clonidina, guanabenz e e } \\
\text { guanfacina. }\end{array}$ & $\begin{array}{l}\text { ALEXANDRE (2008), } \\
\text { BORA et al., (2006), } \\
\text { CORDEIRO (2005), }\end{array}$ \\
\hline
\end{tabular}




\begin{tabular}{|c|c|c|c|c|}
\hline & & & & $\begin{array}{l}\text { COSTA(2006), NICOLLETTI et } \\
\text { al., (2007), OLIVEIRA (2007), } \\
\text { SILVEIRA (2008), SIMÔES } \\
(2007), \text { RDC no 222/2006, } \\
\text { TFOUNI(2007) }\end{array}$ \\
\hline Ginkgo biloba L. & $\begin{array}{l}\text { Ginkgo } \\
\text { biloba }\end{array}$ & Antagonista & $\begin{array}{l}\text { Bloqueadores dos canais de cálcio } \\
\text { Exemplos: Nifedipina, Adalat, Anlodipina }\end{array}$ & $\begin{array}{l}\text { ALEXANDRE (2008), } \\
\text { BARBOSA et al., (2006), } \\
\text { CORDEIRO (2005), COSTA } \\
\text { (2006), NICOLLETTI et al., } \\
\text { (2007), OLIVEIRA (2007), } \\
\text { SILVEIRA (2008), SIMOOES } \\
\text { (2007), RDC no 222/2006, } \\
\text { TFOUNI et al., (2007) }\end{array}$ \\
\hline $\begin{array}{l}\text { Pausinystalia } \\
\text { yohimbe }\end{array}$ & $\begin{array}{l}\text { Casca de } \\
\text { ioimba, } \\
\text { ioimbé }\end{array}$ & Antagonista & $\begin{array}{l}\text { Simpaticolíticos } \\
\text { Exemplos: metildopa, clonidina, guanabenz e } \\
\text { guanfacina. } \\
\text { Inibidores da Enzima conversora de angiotensina } \\
\text { Exemplos: captopril, maleato de enalapril, } \\
\text { lisinopril, ramipril. } \\
\\
375\end{array}$ & $\begin{array}{l}\text { ALEXANDRE (2008), } \\
\text { BARBOSA et al., (2006), } \\
\text { CORDEIRO (2005), COSTA } \\
\text { (2006), NICOLLETTI et al., } \\
\text { (2007), OLIVEIRA (2007), } \\
\text { SILVEIRA (2008), SIMÔES } \\
\text { (2007), RDC no 222/2006, } \\
\text { TFOUNI et al., (2007) }\end{array}$ \\
\hline Ruscus aculeatus L. & Gilbarbeira & Antagonista & $\begin{array}{l}\text { Simpaticolíticos } \\
\text { Exemplos: metildopa, clonidina, guanabenz e } \\
\text { guanfacina. }\end{array}$ & $\begin{array}{l}\text { ALEXANDRE (2008), } \\
\text { BARBOSA et al., (2006), } \\
\text { CORDEIRO (2005), COSTA }\end{array}$ \\
\hline
\end{tabular}




\begin{tabular}{|c|c|c|c|c|}
\hline & & & & $\begin{array}{l}\text { (2006), NICOLLETTI et al., } \\
\text { (2007), OLIVEIRA (2007), } \\
\text { SILVEIRA (2008), SIMOES } \\
\text { (2007), RDC no } 222 / 2006, \\
\text { TFOUNI et al., }(2007)\end{array}$ \\
\hline $\begin{array}{l}\text { Plectranthus barbatus } \\
\text { Andr. }\end{array}$ & Falso Boldo & Antagonista & $\begin{array}{l}\text { Bloqueadores dos canais de Cálcio } \\
\text { Exemplos: Anlodipina, verapamil. }\end{array}$ & $\begin{array}{l}\text { ALEXANDRE (2008), } \\
\text { BARBOSA et al., (2006), } \\
\text { CORDEIRO (2005), COSTA } \\
\text { (2006), NICOLLETTI et al., } \\
\text { (2007), OLIVEIRA (2007), } \\
\text { SILVEIRA (2008), SIMOES } \\
\text { (2007), RDC no } 222 / 2006, \\
\text { TFOUNI et al., (2007) }\end{array}$ \\
\hline Baccharis trimera & Carqueja & Antagonista & $\begin{array}{l}\text { Diuréticos } \\
\text { Exemplos: Furosemida, Hidroclorotiazida } \\
\text {,Clortalidona, Indapamida, } \\
\text { Espironolactona, Amilorida }\end{array}$ & $\begin{array}{l}\text { ALEXANDRE (2008), } \\
\text { BARBOSA et al., (2006), } \\
\text { CORDEIRO (2005), COSTA } \\
\text { (2006), NICOLLETTI et al., } \\
\text { (2007), OLIVEIRA (2007), } \\
\text { SILVEIRA (2008), SIMÕES } \\
\text { (2007), RDC no 222/2006, } \\
\text { TFOUNI et al., (2007) } \\
\end{array}$ \\
\hline
\end{tabular}




\begin{tabular}{|c|c|c|c|c|}
\hline Camellia sinensis (L.) & Chá verde & Antagonista & 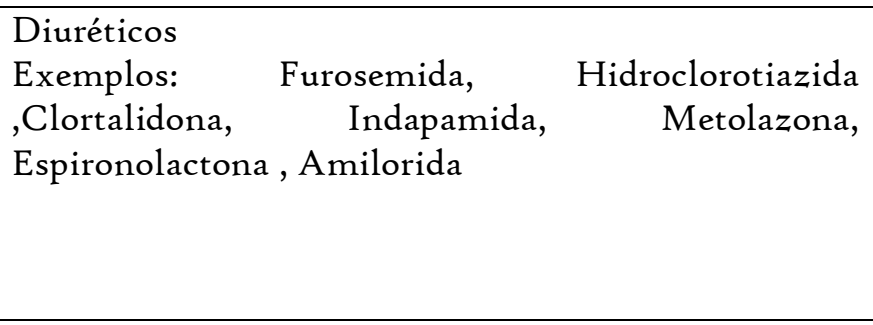 & $\begin{array}{l}\text { ALEXANDRE (2008), BARBOSA et } \\
\text { al., (2006), CORDEIRO (2005), } \\
\text { COSTA (2006), NICOLLETTI et } \\
\text { al., (2007), OLIVEIRA (2007), } \\
\text { SILVEIRA (2008), SIMÔES (2007), } \\
\text { RDC no } 222 / 2006, \text { TFOUNI et al., } \\
(2007)\end{array}$ \\
\hline Hypericum perforatum & $\begin{array}{l}\text { Erva de São } \\
\text { João }\end{array}$ & Antagonista & $\begin{array}{l}\text { Bloqueadores do canais de Cálcio } \\
\text { Exemplos: Anlodipina, verapamil. }\end{array}$ & $\begin{array}{l}\text { ALEXANDRE (2008), BARBOSA et } \\
\text { al., (2006), CORDEIRO (2005), } \\
\text { COSTA (2006), NICOLLETTI et } \\
\text { al., (2007), OLIVEIRA (2007), } \\
\text { SILVEIRA (2008), SIMỐS (2007), } \\
\text { RDC no } 222 / 2006 \\
\text { TFOUNI et al., (2007) }\end{array}$ \\
\hline
\end{tabular}

Fonte : dados da pesquisa (202I)

O controle da hipertensão demanda uma terapia com medicamentos, mas também é importante que a pessoa acometida pela patologia repense sobre seus hábitos de vida, visto que o estilo de vida das pessoas é fator preponderante para a ocorrência da doença (NOGUEIRA, 2014).

O estudo realizado por Rosario et al., (2009) aponta que o manejo de pessoas hipertensas deve basear-se em fatores próprios de cada pessoa, devendo ser avaliada a existência de comorbidades, além da farmacocinética, farmacodinâmica e a idade da pessoa. Rosario et al., (20o9) advertem em sua pesquisa sobre a importância da atenção sobre a terapia prescrita ser redobrada no que diz respeito a pacientes idosos, já que estes possuem por natureza predisposição para a ocorrência de eventos negativos.

Ainda no estudo de Rosario et al., (2009) foi apontado que a principal função dos medicamentos anti-hipertensivos é a redução de níveis pressóricos, bem como o afastamento da ocorrência de eventos cardiovasculares provenientes da elevação da pressão arterial. 
No que diz respeito a utilização de algumas ervas com fins terapêuticos, Castro (2006) em seu texto ressalta que elas acarretam exposição a riscos relevantes na saúde de quem as utiliza de forma inadequada, sobretudo, no contexto da hipertensão arterial sistêmica, o autor indica como principal risco, as interações medicamentosas que podem ocorrer entre medicamentos sintéticos e fitoterápicos.

A pesquisa direcionada por Silveira (2008) destaca que as interações medicamentosas podem ser negativas, ao passo que podem produzir a redução da eficácia de medicamentos, além do aparecimento de reações adversas com variados graus de gravidade.

O autor supracitado adverte em seu estudo que o uso de forma equivocada de plantas medicinais, mesmo aquelas plantas que possuem pouco efeito tóxico, pode desencadear manifestações sérias, sobretudo quando existem fatores de risco associados ao uso, como por exemplo, o uso de medicamentos sintéticos concomitantemente. Silveira adverte ainda sobre a importância da desconstrução do mito perpetuado de que ervas medicinais não acarretam prejuízos à saúde e que elas não produzem efeitos adversos, podendo ser utilizadas sem contraindicação.

Já o estudo de Rocha (2004) indicou a existência de vários motivos que fazem com que as pessoas acreditem que ervas medicinais não trazem danos a saúde, dentre esses motivos estão a dissertação e a consequente publicação de revistas, manuais e livros, sem compromisso com a ciência, disseminando que a utilização de terapias com plantas medicinais são livres de riscos e eficazes para o tratamento de uma gama de patologias, estes materiais apresentam-se, somente com fins de arrecadação do capital.

Barbosa (2008) explica que a interação entre a planta e o medicamento anti-hipertensivo dependerá especialmente do mecanismo de ação de alguns metabólitos secundários.

Neste sentido, Costa (2006) ressalta que os anti-hipertensivos podem ter seus efeitos antagonizados, de modo que ocorre uma modificação do efeito bioquímico ou fisiológico do fármaco pelas plantas que foram elencadas e dispostas no quadro acima, acarretando efeito oposto ao desejado.

De acordo com Oliveira (2007) isto se deve ao fato de que as plantas supracitadas possuem atividade hipertensiva, vasodilatadora e de retenção de líquidos, ou seja, agem de modo oposto aos fármacos anti-hipertensivos.

No que diz respeito a interação dos anti-hipertensivos das classes denominados Simpaticolíticos(metildopa) e Inibidores da ECA ramipril (marca: Altace), captopril (Capoten), enalopril (Vasotec), fosinopril (Monopril), lisinopril (Prinivil, Zestril) e quinapril (Accupril) com a planta Pausinystalia yohimbe mais conhecida popularmente como Casca de ioimba, ioimbé, Nicolletti et al., (2007) explica que a interação acontece porque a planta possui um alcalóide conhecido como ioimbina que possui um potencial antagônico sobre alfa-2-adrenoreceptor, desta forma, promove a interação com anti-hipertensivos das classes dos Simpaticolíticos e Inibidores da ECA, pois estes medicamentos atuam no mesmo sítio, antagonizando seus efeitos e consequentemente elevando a pressão arterial.

O mesmo ocorre com a erva Catha edulis, Simões (2007) relata que estas ervas interagem com a classe dos Simpaticolíticos, antagonizando os efeitos destes medicamentos, aumento a pressão arterial das pessoas que as utilizam ao mesmo tempo em que estão fazendo uso da planta citada.A erva Ruscus aculeatus, demonstrada na, é descrita por Silveira (2008) como uma planta que interage com a classe de anti-hipertensivos chamada de Simpatolíticos, a interação acontece porque essa planta age no mesmo sitio de ação dos 
medicamentos Simpatolíticos, sendo assim antagonizando o efeito do medicamento e aumenta a pressão arterial.

A planta Ginkgo biloba L., pode aumentar a frequência de efeitos adversos dos antihipertensivos das classes bloqueadores dos canais de cálcio e diuréticos, tais como cefaléia, rubor e edema de tornozelo. Quando administramos o Ginkgo biloba associado a diuréticos tiazídicos(hidroclorotiazida) pode provocar, após uma semana, o aumento da pressão sanguínea do paciente, podendo reverter o quadro com a suspensão das duas medicações. Quando administramos também o Ginkgobilobacom anti-hipertensivos inibidores dos canais de cálcio, como nifedipina, anlodipina e diltiazem, podemos fazer com que haja um aumento nos efeitos adversos destes fármacos (ALEXANDRE, 2008).

Das plantas citadas, o Plectranthus barbatus Andr., é a mais conhecida popularmente, a interação medicamentosa destas plantas com os anti-hipertensivos ditos bloqueadores dos canais de cálcio acontece devido a ocorrência de ações inotrópicas negativa, levando à diminuição da sua excitabilidade e contractilidade e consequentemente elevação da pressão arterial (CORDEIRO e CHUNG, 2005).

No que diz respeito a planta Hypericum perforatum, Cordeiro (2005) explica que a interação medicamentosa ocorre entre a planta e classe de anti-hipertensivo bloqueadores dos canais de cálcio devido ao mecanismo de ação da planta, onde esta induz a isoforma $\mathrm{CYP}_{3} \mathrm{~A}_{4}$, visto que os bloqueadores dos canais de cálcio são substratos para a isoforma $\mathrm{CYP}_{3} \mathrm{~A}_{4}$, aumentando a velocidade de metabolização pela $\mathrm{CYP}_{3} \mathrm{~A}_{4}$, geralmente acelerando sua eliminação do organismo (ou raramente acelerando sua ativação no caso de prófármacos). Assim, os efeitos dos medicamentos metabolizados por $\mathrm{CYP}_{3} \mathrm{~A}_{4}$ duram menos e exigem doses maiores.

A Baccharis trimera, também é uma planta medicinal que apresenta contra indicação de sua utilização concomitante com anti-hipertensivos, sobretudo, com a classe dos bloqueadores beta adrenérgicos. Barbosa et al., (2006) adverte que esta planta interage com os bloqueadores beta adrenérgicos, pois este tipo de medicamento é metabolizado no fígado e a Baccharis trimera, tem a capacidade de reduzir o efeito terapêutico de drogas que fazem metabolismo hepático.

De acordo com Barbosa et al., (2006) e com o levantamento bibliográfico aqui realizado, as interações entre os medicamentos anti-hipertensivos e as substâncias existentes nas plantas medicinais ocorrem, o que colabora para a desconstrução de que as plantas não acarretam prejuízos a saúde, podendo ser utilizadas indiscriminadamente.

A Resolução no 477/2008 do Conselho Federal de Farmácia, atribui ao profissional Farmacêutico, além de outras ações a promoção do uso racional de plantas medicinais, através de divulgação e comunicação aos pacientes, contribuindo para que as pessoas façam o uso racional de plantas medicinais.

Para Jesus et al., (2013) a promoção do uso racional de ervas medicinais realizada pelo Farmacêutico é uma prática importante, visto que utiliza-las de forma irracional acarreta danos à saúde dos indivíduos, pode propiciar um maior tempo de tratamento e cura, aumentar os custos, além de provocar interações medicamentosas negativas.

A assistência farmacêutica, de acordo com Fernandes (2015) consiste em papel relevante quando se trata dos serviços de atenção à saúde, já que traz como premissa promover o uso racional de medicamentos, incluindo práticas que envolvem a escolha da terapia mais adequada, que garanta a menor ocorrência de efeitos adversos, avaliação das 
contraindicações existentes, garantia de que o paciente faça a adesão da terapia medicamentosa, o que garantirá, sobretudo, a segurança na utilização de plantas medicinais.

É importante destacar que o uso de ervas medicinais, embora se perpetue o mito de que não acarretam riscos a saúde por serem de origem vegetal, podem sim, acarretar risco à saúde quando são usadas sem orientação de um profissional habilitado (NICOLETTI et al., 2010).

Desta forma, Diaz (2009) alerta sobre a necessidade de que ao prestar a assistência farmacêutica, o farmacêutico pesquise se o paciente faz uso de algum outro medicamento, evitando que ocorram as interações medicamentosas, que podem provocar a redução do efeito ou a potencialização do efeito fazendo com que a terapia medicamentosa não atinja o objetivo desejado.

Além disso, Diaz (2009) destaca a necessidade da identificação de grupos de risco, no caso deste trabalho os paciente hipertensos, é importante também analisar a prescrição feita pelo médico, com vistas a identificar a ocorrência de prescrição indevida e utilização inadequada (NICOLETTI et al., 2010).

É atribuição do farmacêutico ainda, a orientação do paciente sobre o uso apropriado de plantas medicinais, os possíveis eventos adversos, sobretudo, quando o uso é realizado concomitantemente com outros fármacos.

A Resolução nº 546/20II disserta que o farmacêutico pode indicar plantas medicinais quando o paciente solicita, a indicação deverá ser feita de maneira clara, tendo registro em documento e uma via ser entregue ao paciente e a outra via deverá ficar no poder do farmacêutico.

Com a Resolução citada, o farmacêutico tem na sua indicação pautada na prevenção de problemas ligados ao uso de medicamentos, a sensibilização do paciente em aderir a terapia medicamentosa, monitoramento e avaliação da resposta da terapia proposta, bem como a aproximação do farmacêutico na comunidade.

\section{CONSIDERAÇÕES FINAIS}

Neste estudo, evidenciou-se que o uso de plantas medicinais é algo antigo, e que por vezes se configura em uma única terapia para a população, visto que são de baixo custo, além da perpetuação da ideia de que as plantas por serem de origem vegetal, não apresentam nocividade a vida das pessoas, sendo mais seguras que fármacos sintéticos, outro fator que pode estar relacionado ao aumento do consumo das ervas e a deficiência no acesso aos serviços de saúde. Sendo assim, são utilizadas de forma irracional, sem a orientação de um profissional habilitado, acarretando risco à saúde, que podem propiciar a ocorrência de eventos adversos e interações medicamentosas negativas se utilizados concomitantemente com outros medicamentos.

Neste sentido, destaca-se a importância do papel do profissional farmacêutico neste contexto, visto que a sua atuação está implicada na minimização da ocorrência do uso racional de medicamentos e plantas medicinais para que diminua os riscos de interações medicamentosas com anti-hipertensivos e alteração na pressão arterial.

\section{REFERÊNCIAS}


AHMAD, L. Ethnopharmacological documentation of medicinal plants used for hypertension among the local communities of DIR Lower, Pakistan. Journal of ethnopharmacology, vol. 175, p. 58-66, 2015.

ALEXANDRE, R.F.; Bagatini, F.; Simões, C. M. O. Interações entre fármacos e medicamentos fitoterápicos à base de ginkgo ou ginseng. Revista Brasileira de Farmacognosia, vol 18, p. 117-126, 2008.

BADKE, M.R.; BUDO, M.L.D.; ALVIM, N.A.T.; ZANETTI, G.D.; HEISLER, E.V. Saberes e práticas populares de cuidado em saúde com o uso de Plantas Medicinais. Texto Contexto Enfermagem, p. 363-370, 2012.

BARBOSA-FILHO, J.M.; MARTINS, V.K.M.; RABELO, L.A.; MOURA, M.D.; Natural products inhibitors of the angiotensin converting enzyme (ACE). A review between 19802000. Revista Brasileira de Farmacognosia, p. 421-446, 2008.

CASTRO, D.L.L. Aspectos toxicológicos das plantas medicinais utilizadas no Brasil: um enfoque qualitativo no Distrito federal. Pós-graduação Latu Sensu. Curso de Especialização em qualidade em alimentos. Universidade de Brasília CET-Centro de Excelência em Turismo, Brasília-DF, 2006.

CORDEIRO CHG, CHUNG MC, SACRAMENTO LVS 2005. Interações medicamentosas de fitoterápicos e fármacos: Hypericum perforatum e Piper methysticum. Rev Bras Farmacogn (15), p. 272-278.

COSTA, M.C.C.D. Uso popular e ações farmacológicas de Plectranthus barbatus Andr. (Lamiaceae): revisão dos trabalhos publicados de 1970 a 2003. Rev Bras Pl Med.,. vol. 8, n. 2, p. 8I-88, 2006.

DIAS, MG. Interações entre preparações á base de plantas medicinais e medicamentos. Rev. de Fitoterapia. vol. 9, p. 5-22, 2009.

FERNANDES, W. S.; Cembranelli, J. C. S. Automedicação e o uso irracional de medicamentos. O papel do profissional farmacêutico no combate a essas práticas. Revista UniVap , v. 2I, p. 5, 2015.

JESUS, A. P. G. A. S.; YOSHIDA, N. C. P.; FREITAS, J. G. A. Prevalência da automedicação entre acadêmicos de farmácia, medicina, enfermagem e odontologia. Rev. Eletrônica de Farmácia, v. 40, n. 2, 2013.

MACIEL, M. A. M.; PINTO, A. C.; JR, V. F. V. Plantas medicinais: a necessidade de estudos multidisciplinares. Quim. Nova, Vol. 25, No. 3, p. 429-438, 2012.

NiCOletTi, M. A.; CARVAlhO K. C.; OliveirA JR, M. A.; BeRTASSO, C. C.; CAPOROSSI, P. Y.; TAVARES, A. P. L. L Uso popular de medicamentos contendo drog as 
de origem vegetal e/ou plantas medicinais: principais interações decorrentes. Revista saúde (UnG. Online), v. 4, n. I, p. 25-39, 2010.

NOGUEIRA, D.; FAERSTEIN, E.; COELI, C. M.; CHOR, D.; LOPES, C. S.; WERNECK, G. L. Reconhecimento, tratamento e controle da hipertensão arterial: estudo Pró-Saúde, Brasil. Rev. Panam Salud Publica, Washington, v. 27, n. 2, p. I03-I09, 2014.

OlIVEIRA, C. J. de; ARAÚJO, T. L. de. Plantas medicinais: usos e crenças de idosos portadores de hipertensão arterial. Revista Eletrônica de Enfermagem. v.9, n.I, p. 93-Io5, 2007.

RAWAT, P. Anti-hypertensive medicinal plants and their mode of action. Journal of Herbal Medicine, v. 6, p. I07-II8, 2016.

Resolução no 477 , de 28 de maio de 2008. Dispõe sobre as atribuições do farmacêutico no âmbito das plantas medicinais e fitoterápicos e dá outras providências.

Resolução no 546, de 2I de julho de 20Ir. Dispõe sobre a indicação farmacêutica de plantas medicinais e fitoterápicos isentos de prescrição e o seu registro.

Resolução RE n. 89, de I6 de março de 2004. Dispõe sobre a lista de registro simplificado de fitoterápicos.

Resolução RDC no 222, de 28 de dezembro de 2006a. Dispõe sobre os procedimentos de petição e arrecadação eletrônica no âmbito da Agência Nacional de Vigilância Sanitária ANVISA e de suas Coordenações Estaduais e Municipais de Vigilância Sanitária e dá outras providências. Diário Oficial da União, Brasília, DF, 29 dez. 2006

ROCHA, F. A. G da. Diagnóstico sobre o uso de plantas medicinais comercializadas no mercado central da Cidade de Mossoró. Monografia. UERN. Mossoró-RN, abr. 2004.

ROSARIO TM, Scala LCNS, Franca GVA, Pereira MRG, Jardim PCBV. Prevalência, controle e tratamento da hipertensão arterial sistêmica em Nobres, MT. Arq Bras Card. 2009; 93: $672-8$.

SILVEIRA, P. F da; BANDEIRA, M. A. M; ARRAIS, P. S. D. Farmacovigilância e reações adversas às plantas medicinais e fitoterápicos: uma realidade. Revista Brasileira de Farmacognosia. v. I8, n.4, p. 618-626, 2008.

SIMÕES, C.M.O.; ALEXANDRE, R.F; BAGATINI F, (Orgs.). Farmacognosia: da planta ao medicamento. 6.ed. revisada e ampliada. Porto Alegre/Florianópolis: Editora da UFRGS/Editora da UFSC, p. 13-26, 2007. 
Sociedade Brasileira de Cardiologia / Sociedade Brasileira de Hipertensão/Sociedade Brasileira de Nefrologia. VI Diretrizes Brasileiras de Hipertensão. Rio de Janeiro: Arq Bras Cardiol, v. 95, n. I, supl.ı, p. I-5I, 2010.

TFOUNI, Sílvia Amélia Verdiani et al . Contribuição do guaraná em pó (Paullinia cupana) como fonte de cafeína na dieta. Rev. Nutr., Campinas, v. 20, n. I, p. 63-68, 2007. 